\title{
O pré-natal do parceiro sexual: importância para a saúde do homem e da gestante
}

The prenatal of the sexual partner: importance for the health of men and pregnant women

El prenatal de la pareja sexual: importancia para la salud de hombres y mujeres embarazadas

Felipe Mateus Vitoretti ${ }^{1}$, Lucas Gonçalves Soares Drummond Penna ${ }^{1}$, Matheus Gabriel Santos Souza ${ }^{1}$, Miguel de Assis Simões Couto ${ }^{1}$, Rafael Simão e Silva², Leda Caldeira de Souza², Marcelo Brandão Jacob², Sâmara Pereira de Miranda², Marcela Rodrigues Machado², Juliana Barroso Zimmermmann ${ }^{1,2 *}$.

\section{RESUMO}

Objetivo: Avaliar a saúde dos parceiros sexuais de gestantes, bem como a sua importância para a evolução da gestação. Métodos: Trata-se de um estudo transversal, realizado com 81 indivíduos. Foram analisados parceiros participativos à consulta até 32 semanas de gestação, submetidos a anamnese, pesquisa de antecedentes pessoais, familiares, participação na gravidez da parceira, exame físico e exames laboratoriais; dados e intercorrências clínicas durante o pré-natal das gestantes acompanhas pelo parceiro foram colhidos, utilizou como grupo controle gestantes com pré-natal sem participação de parceiros. Estatísticas foram realizadas com software JAMOVI (ios) versão 1.1.9. O estudo foi aprovado pelo Comitê de Ética em Pesquisa. Resultados: Considerando a participação do parceiro ou não durante o pré-natal, não houve mudanças estatisticas significativas em relação a progressão esperada da gestação bem como as complicaçoes mais comuns dessa fase. Em relação a saude do parceiro o fator de maior preucupação foi a média de IMC classificada como sobrepeso. Conclusão: A participação do parceiro no pré-natal, não influenciou nas intercorrências clínicas da gestante para o determinismo do parto, mas foi possível identificar alterações da própria saúde do parceiro que merecem orientação, controle e tratamento.

Palavras-chave: Cuidado pré-natal, Paternidade, Gestantes.

\begin{abstract}
Objective: Evaluate the health of the sexual partners of pregnant women, as well as its importance for the evolution of pregnancy. Methods: Cross-sectional study, carried out with 81 individuals. Partners that participated in consultation up to 32 weeks of gestation were submitted to anamnesis, research of personal and family history, participation in the partner's pregnancy, physical examination and laboratory tests; data and clinical complications during the prenatal care of pregnant women accompanied by the partner were collected. The control group, pregnant women without the participation of partners in the prenatal care, was evaluated using a previous database. Statistics were performed with JAMOVI software (ios) v1.1.9. The study was approved by the Research Ethics Committee. Results: The participation of the partner or not during prenatal care showed no statistically significant changes in relation to the expected progression of pregnancy or the most common complications. Regarding the partner's health, the factor of greatest concern was the average BMI (overweight). Conclusion: The partner's participation in prenatal care did not influence the clinical complications of the pregnant woman for the determinism of delivery, but it was possible to identify changes in the partner's own health that deserve, guidance, control and treatment.
\end{abstract}

Keywords: Prenatal care, Paternity, Pregnant women.

${ }^{1}$ Faculdade de Medicina de Barbacena, Barbacena - MG. *E-mail: julianabz@uol.com.br

${ }^{2}$ Faculdade de Medicina da Universidade Federal de Juiz de Fora/Hospital Universitário, Juiz de Fora - MG. 


\section{RESUMEN}

Objetivo: Evaluar la salud de las parejas sexuales de gestantes y su importancia para la evolución del embarazo. Métodos: Estudio transversal, realizado con 81 individuos. Las parejas participantes fueron consultadas hasta las 32 semanas de gestación, sometidas a anamnesis, antecedentes personales, antecedentes familiares, participación en el embarazo de la pareja, exploración física y pruebas de laboratorio; Se recolectaron datos y complicaciones clínicas durante el cuidado prenatal de gestantes acompañadas por la pareja. El grupo control, gestantes con atención prenatal sin participación de pareja, fue evaluado en una base de datos previa. Estadísticas: software JAMOVI (ios) v1.1.9. Aprobado por el Comité de Ética en Investigación. Resultados: Considerando la participación o no de la pareja durante la atención prenatal, no hubo cambios estadísticamente significativos en relación a la progresión esperada del embarazo, así como las complicaciones más comunes. En cuanto a la salud de la pareja, el factor de mayor preocupación fue el IMC promedio (sobrepeso). Conclusión: La participación de la pareja en la atención prenatal no influyó en las complicaciones clínicas de la gestante para el determinismo del parto, pero sí fue posible identificar cambios en la propia salud de la pareja que merecen orientación, control y tratamiento.

Palabras clave: Atención prenatal, Paternidad, Mujeres embarazadas.

\section{INTRODUÇÃO}

Na família atual, o homem já não é mais o único provedor. Várias mudanças culturais colaboraram para que os papéis de pai e mãe na família fossem modificados, o que permitiu que os homens pudessem vivenciar a paternidade de forma mais afetiva (FREITAS WMF, et al., 2009). Entretanto, no Brasil, os serviços de saúde são desenvolvidos para o acolhimento da mulher gestante, mas não para o acolhimento do pai.

Desta forma, os pais costumam ser excluídos neste atendimento e isso pode ser explicado pela pouca infraestrutura física, ausência de capacitações e descrédito dos profissionais sobre esse público em relação aos homens de classes mais baixas (CARDOSO VEPS, et al., 2018).

Outro fator importante é o fato de os homens terem maior dificuldade em reconhecer suas necessidades de saúde, rejeitando a possibilidade de adoecer (COFEN, 2017). Isto faz com que acabem procurando o sistema de saúde através da assistência especializada, tendo como consequência o agravo das doenças, muitas vezes, pela demora no atendimento (MINISTÉRIO DA SAÚDE, 2016).

Conhecendo esse problema, o Ministério da Saúde implementou a Política Nacional de Atenção Integral à Saúde do Homem que visa o autocuidado masculino, mas também o entendimento universal que a saúde é um direito dos homens brasileiros. Muitas pesquisas demonstram que os homens são mais vulneráveis às doenças, morrem mais cedo que as mulheres e apresentam um ponto em comum, não buscam a assistência básica. Além disso, não são adeptos aos tratamentos longos, como é necessário em muitas doenças crônicas e as mudanças de hábitos e comportamentos não são cumpridas, na maioria das vezes (MINISTÉRIO DA SAÚDE, 2008). Ademais, variáveis culturais são também responsáveis pelo descuido dos homens com sua própria saúde, já que a doença determina uma fragilidade que habitualmente o homem não quer estar associado (FREITAS WMF, et al., 2009; MINISTÉRIO DA SAÚDE, 2016).

Pode-se dizer que a gestação independente da configuração familiar, traz à tona uma série de emoções e de decisões que impactam o cotidiano das já formadas ou futuras famílias. Nesse sentido, o período da gestação é de grande importância para todas as pessoas envolvidas para que as mudanças na rotina e as adaptações decorrentes da chegada desta nova vida, transcorram de forma tranquila para todos. No entanto, para muitos homens, o período da gestação pode suscitar nas mais diversas emoções e até mesmo em sintomas físicos e, por isso, eles também merecem atenção do serviço de saúde. Não são raros os casos de cefaléia, alteração dos hábitos alimentares, insônia, taquicardia e fadiga na vigência de um estresse e o nascimento de um filho pode ser considerado um estressor para os envolvidos no processo (MARGIS R, et al., 2003; HENZ GS, et al., 2017; FARIAS SMC, et al., 2011).

O Ministério da Saúde (MS) vem estimulando o pré-natal do parceiro desde 2011, para que esse possa fazer parte do processo gestacional, sentindo-se pai desde o pré-natal. Muitos homens acreditam que se 
tornam pais, apenas após o nascimento do bebê. Essa iniciativa estimula a paternidade responsável, cuidadora e responsável, aumentando o elo entre pai e filho (a).

A consulta pré-natal do parceiro foi incluída no rol do Sistema Único de Saúde e permite avaliar as suas condições de saúde. Para isso, o MS estimulou a realização de exames complementares laboratoriais, testes rápidos e atualização do cartão de vacinas, além de incluir as orientações sobre a gravidez, parto, puerpério, amamentação e seus direitos como pai/parceiro (FREITAS WMF, et al., 2009; CORTEZ MB, et al., 2016; BENAZZI AST, et al., 2011; DUARTE G, 2007).

O pré-natal do parceiro visa não apenas a integração do homem no cuidado pré-natal, mas também uma oportunidade de inserir o homem nas ações voltados para o cuidado integral da sua própria saúde. Sendo assim, prática de atividades físicas, alimentação balanceada e saudável, redução do consumo de bebidas alcoólicas e deixar de fumar são algumas orientações na abordagem do homem. O parceiro recebe também orientações relativas aos direitos da gestante e aos seus próprios direitos.

Estudo realizado por Holanda SM, et al. (2018) identificou que a participação do parceiro no pré-natal influencia também no parto. Nesse estudo, os parceiros compareceram a pelo menos duas consultas de prénatal e isso influenciou diretamente na compreensão e entendimento de seu papel no mecanismo da gestação, parto e puerpério. Outro estudo, identificou que a participação do parceiro no parto, melhora sua qualidade e influencia positivamente na compreensão e aceitação das orientações no puerpério (AGUIAR C e JENNINGS L, 2012).

O fato é que o pré-natal do parceiro reduz violência doméstica, a possibilidade de depressão puerperal, aumenta os laços afetivos, além de proporcionar detecção precoce de infecções sexualmente transmissíveis, especialmente, a sífilis (HORTA HHL, et al., 2017). Além disso, a participação do parceiro no pré-natal favorece a adesão da gestante, melhorando os indicadores de saúde perinatais (FABIO SV, 2016).

Outro dado interessante é que a participação do pai no pré-natal pode variar com a idade, escolaridade, com o planejamento da gravidez e com o número de filhos. Assim, gestações planejadas, do primeiro filho, com pais adultos (entre 25 e 40 anos de idade), estão mais associadas ao envolvimento paterno em todo 0 processo gestacional (NOGUEIRA JDF e FERREIRA M, 2008).

Apesar de lei, vemos que muitas unidades de saúde não estão preparadas para esse atendimento, seja por falta de equipe, de material ou de recursos financeiros disponíveis para a realização das avaliações dos parceiros e da demanda dos exames complementares. O objetivo do estudo foi avaliar a saúde dos parceiros sexuais e a importância dessa participação na saúde das gestantes atendidas nos serviços de Obstetrícia da Faculdade de Medicina de Barbacena e da Universidade Federal de Juiz de Fora (UFJF), bem como a sua importância para a evolução da gestação.

\section{MÉTODOS}

Foram estudados gestantes e seus parceiros sexuais que compareceram espontaneamente à consulta na qualidade de acompanhantes até 32 semanas de gestação de sua parceira. Foram excluídos aqueles que não desejaram participar do estudo, ou aqueles que só compareceram à consulta após 32 semanas de gestação, pela impossibilidade de termos os exames em tempo hábil para a avaliação.

Esses homens foram submetidos a anamnese e pesquisa de antecedentes pessoais e familiares (idade, número de filhos, filhos de outros relacionamentos, doenças de base, tratamentos prévios, cirurgias, uso de medicação, tabagismo, alcoolismo), bem como a sua participação na gravidez da companheira (planejamento da gravidez, desejo de ser pai, condições econômicas, participação no pré-natal, ajuda nas tarefas diárias). A seguir, foi realizado o exame físico com avaliação das mucosas (coloração, hidratação), peso, altura, IMC, circunferência abdominal e pesquisa de edemas (PORTO CC e PORTO AL, 2019).

A seguir, foram solicitados exames - a propedêutica do parceiro sexual, definida no estudo: hemograma, VDRL, grupo sanguíneo ABO e Rh, HBsAg, anti HCV, anti HIV, colesterol total, HDL, triglicérides e glicemia de jejum. Os parceiros foram avaliados em uma única consulta, com agendamento para avaliação dos exames 
complementares. Receberam ainda orientação sobre seus resultados, com informação pré e pós teste e orientação sobre estilo de vida saudável, revisão da caderneta de vacinas e atendimento especializado quando necessário ao caso (MINISTÉRIO DA SAÚDE, 2016).

Como desfechos primários foram considerados o estado de saúde dos homens (hipertensão arterial crônica, obesidade, diabetes, hipercolesterolemia, hipertrigliceridemia, ansiedade, tabagismo, alcoolismo). Como desfechos secundários foram considerados o estado de saúde das gestantes (pré-eclâmpsia, diabetes gestacional, hiperemese gravídica, ansiedade, trabalho de parto prematuro, amniorrexe prematura e DPP). Como controle dessas gestantes, foram utilizados um grupo de gestantes previamente selecionadas que fizeram pré-natal sem a participação dos parceiros e que já se encontra em nosso banco de dados. Todas essas gestantes (controles) concordaram em participar dos estudos e cederam seus dados para a participação em trabalhos científicos.

Os estudos sobre o pré-natal do parceiro são incipientes no Brasil, com amostragem pequenas e metodologia qualitativa. Para tanto, realizou-se uma amostragem de conveniência para este estudo de 50 homens e 100 gestantes, sendo 50 gestantes com participação do parceiro e 50 gestantes sem a participação do parceiro sexual. Considerando um estudo piloto, a quantidade necessária para uma avaliação inicial seria de 20 parceiros e 40 gestantes, sendo $20 \mathrm{com}$ parceiros que participaram do pré-natal e 20 sem parceiros participantes do pré-natal.

Os dados foram digitalizados em formulários eletrônicos de banco de dados Access desenvolvidos especialmente para os estudos. Os resultados obtidos foram expressos como média e desvio padrão para variáveis quantitativas e como frequência absoluta e frequência relativa para as variáveis categóricas. As análises estatísticas foram realizadas com auxílio do software JAMOVI (ios) versão 1.1.9. Utilizou-se o teste t de Student para comparação de médias entre amostras independentes. Para verificar associação, utilizouse $o$ teste exato de Fischer quando possível ou o teste do $x 2$ para variáveis com mais de duas categorias. 0 nível de significância adotado foi de $5 \%$. O presente estudo foi encaminhado à Comissão de Ética e aprovado para ser realizado.

\section{RESULTADOS}

Foram estudadas 49 gestantes e 32 parceiros. A média de idade das gestantes foi de 33,7 +5,14 anos. Em relação ao número de gestações, partos e abortos, a média foi de 1,92 + 1,35 gestações, 0,72 + 1,14 partos e 0,24 + 0,59 abortos. A pressão arterial média das gestantes foi de $90,7+10,5 \mathrm{mmHg}$ e a idade gestacional média no início do pré-natal 10,2+3,21 semanas de gestação. Realizou-se a comparação entre as médias das idades, partos, gestações e abortos, considerando a participação do parceiro ou não no prénatal, mas não houve significância estatística. As médias foram comparadas para cada grupo, considerando $\mathrm{p}<0,05$ (Tabela 1).

Tabela 1 - Aspectos epidemiológicos das pacientes estudadas considerando a participação do parceiro no pré-natal.

\begin{tabular}{lcccccc}
\hline \multirow{2}{*}{$\begin{array}{l}\text { Dados } \\
\text { analisados }\end{array}$} & $\begin{array}{c}\text { Parceiro no } \\
\text { pré-natal }\end{array}$ & Número & $\begin{array}{c}\text { Média } \\
\text { Idade }\end{array}$ & $\begin{array}{c}\text { Média } \\
\text { Gesta }\end{array}$ & $\begin{array}{c}\text { Média } \\
\text { Para }\end{array}$ & $\begin{array}{c}\text { Média } \\
\text { Aborto }\end{array}$ \\
\cline { 2 - 7 } & Sim & 32 & 32,9 & 1,94 & 0,781 & 0,188 \\
& Não & 17 & 34,9 & 1,82 & 0,588 & 0,353 \\
\hline & Valor de $\mathbf{p}$ & & 0,193 & 0,781 & 0,577 & 0,361 \\
\hline
\end{tabular}

Fonte: Vitoretti FM, et al., 2020.

Foi também avaliada a idade gestacional no parto, entretanto, as médias foram similares nos dois grupos (38,3 semanas $\times 38,2$ semanas; $p=0,877)$. $O$ tipo de parto não se mostrou diferente $(p=0,10)$, de forma que a cesariana foi a via de parto mais frequente em ambos os grupos. Destaca-se que $61,2 \%$ das pacientes não apresentaram qualquer complicação, mas o diabetes mellitus gestacional (DMG) foi a mais frequente $(22,4 \%)$ (Figura 1). 
Figura 1 - Frequência das principais complicações identificadas no pré-natal.

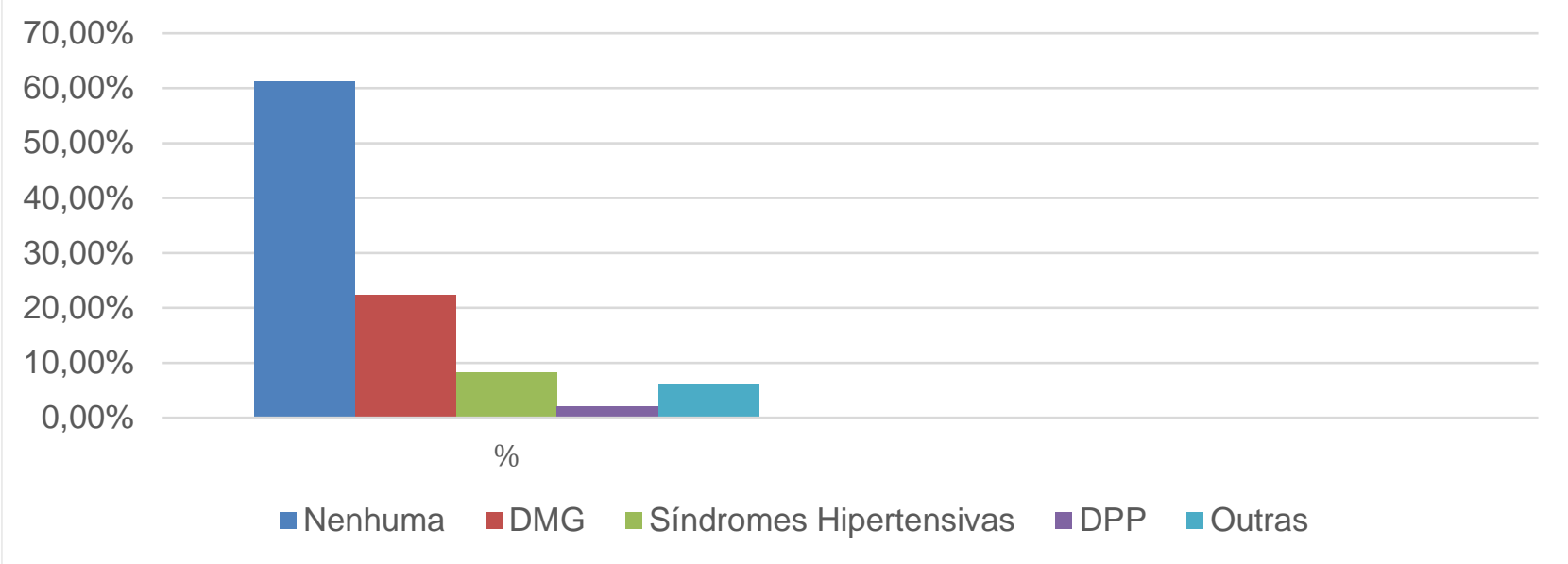

Fonte: Vitoretti FM, et al., 2020.

As complicações identificadas no pré-natal foram comparadas com a presença ou não do parceiro durante pré-natal, mas não houve associação estatística $(p=0,441)$ (Tabela 2).

Tabela 2 - Associação entre complicações identificadas nas gestantes durante o pré-natal e participação do parceiro.

\begin{tabular}{lccc}
\hline \multirow{2}{*}{ Complicações } & \multicolumn{2}{c}{ Parceiro no Pré-natal } & \multirow{2}{*}{ Valor de $\mathbf{p}$} \\
\cline { 2 - 3 } identificadas no pré-natal & Sim & Não & \\
\cline { 2 - 3 } Nenhuma & 21 & 9 & \\
DMG & 6 & 5 & 0,441 \\
Síndrome hipertensivas & 3 & 1 & \\
DPP & 0 & 1 & \\
Outras & 0 & 3 & \\
\hline
\end{tabular}

Fonte: Vitoretti FM, et al., 2020.

A grande maioria das pacientes não apresentou complicações que influenciaram no determinismo do parto. A associação destas complicações com a participação do parceiro no pré-natal foi também testada, mas também não houve significância estatística (Tabela 3).

Tabela 3 - Complicações identificadas nas gestantes para o determinismo do parto e a participação do parceiro no pré-natal.

\begin{tabular}{lccc}
\hline \multirow{2}{*}{$\begin{array}{c}\text { Complicações } \\
\text { identificadas para }\end{array}$} & \multicolumn{2}{c}{ Parceiro no Pré-natal } & Valor de $\mathbf{p}$ \\
\cline { 2 - 3 } o determinismo do parto & Sim & Não & \\
\hline Nenhuma & 20 & 12 & 0,795 \\
DMG & 7 & 2 & \\
Síndrome hipertensivas & 2 & 1 & \\
Outras & 2 & 3 & \\
\hline
\end{tabular}

Fonte: Vitoretti FM, et al., 2020.

Quando se avaliou o estado de saúde dos parceiros, identificou-se que a grande maioria não era fumante (98,3\%), 100\% declararam etilismo social e $59,7 \%$ declararam-se tranquilos, sem qualquer ansiedade em relação à mudança familiar pela gravidez (Tabela 4). 
Tabela 4 - Tabagismo, etilismo, ansiedade e doença de base dos parceiros estudados.

\begin{tabular}{|c|c|}
\hline Dados clínicos / epidemiológicos dos parceiros sexuais & $\mathbf{N}$ \\
\hline Tabagismo & \\
\hline Sim & 2 \\
\hline Não & 30 \\
\hline Álcool & \\
\hline Social & 32 \\
\hline Ansiedade & \\
\hline Sim & 9 \\
\hline Não & 14 \\
\hline Não sabe & 9 \\
\hline Doença de base & \\
\hline Nenhuma & 29 \\
\hline HAC & 1 \\
\hline Tireoidite & 1 \\
\hline LES & 1 \\
\hline
\end{tabular}

Fonte: Vitoretti FM, et al., 2020.

No exame físico dos parceiros sexuais identificou-se sobrepeso. A média de idade foi de 34,9+21 anos. Não houve nenhum caso de sífilis, HIV ou hepatite B e C dentre os parceiros estudados e o lipidrograma já mostra níveis de colesterol e triglicérides acima dos valores ideais (Tabela 5).

Tabela 5 - Dados clínicos dos parceiros estudados.

\begin{tabular}{lllllll}
\hline Dados clínicos & PAS & PAD & CA & Altura & Peso & IMC \\
\hline Média & 119 & 76,3 & 92,8 & 1,73 & 84,2 & 28,2 \\
Mediana & 120 & 80 & 92 & 1,72 & 88 & 29,2 \\
Desvio-padrão & 14 & 10,4 & 10,3 & 0,058 & 12 & 3,43 \\
Mínimo & 100 & 60 & 70 & 1,63 & 60,5 & 22,1 \\
Máximo & 170 & 100 & 107 & 1,90 & 110 & 33,9 \\
\hline Dados laboratoriais & Hb & HTC & Glicemia & Colesterol Total & Triglicérides & HDL \\
\hline Média & 15 & 46 & 86,9 & 204 & 167 & 49,4 \\
Mediana & 15 & 46 & 85 & 197 & 162 & 50,5 \\
Desvio-padrão & 1,41 & 4,24 & 9,91 & 37,1 & 85,4 & 17,5 \\
Mínimo & 14 & 43 & 74 & 155 & 45 & 24 \\
Máximo & 16 & 49 & 100 & 261 & 341 & 74
\end{tabular}

Legenda: IMC (índice de massa corporal); CAPARCEIRO (circunferência abdominal); PASPARCEIRO (pressão arterial sistólica), PADPARCEIRO (pressão arterial diastólica); Hb (hemoglobina (mg/dl); HTC hematócrito (\%); glicemia (mg/dl); colesterol total (mg/dl), triglicérides (mg/dl); HDL 9mg/dl).

Fonte: Vitoretti FM, et al., 2020.

\section{DISCUSSÃO}

A relevância do pré-natal do pai/parceiro está no desenvolvimento da tríade mãe/pai/filho, tornando seu estado de saúde um elemento de preocupação para o bem-estar da família, como um todo. A participação no pré-natal, realizando os exames requisitados e abordando as alterações encontradas, indiretamente influencia no resguardo da saúde física, emocional e financeira da criança.

Importante ressaltar que muitas mulheres consideram o momento do pré-natal um espaço destinado apenas a avaliação feminina e do bebê e, sendo assim, excluem o marido/companheiro desse momento. A orientação de que gestar é uma tarefa do casal e que a participação masculina permite a criação de vínculo paterno de forma mais precoce deve fazer parte da rotina pré-natal e estimulada pelo médico assistente (CARDOSO VEPS, et al., 2018).

É válido ressaltar que a presença do parceiro durante as consultas de pré-natal pode ser uma oportunidade única de aprendizado para eles, já que muitos tem uma elevada carga de ansiedade provocada pelo desconhecimento. Neste estudo, $28 \%$ declararam-se ansiosos com a formação da nova família. Isso é 
interessante porque esta avaliação foi realizada apenas através de pergunta habitual (Como você se sente com a formação da nova família?), não tendo sido realizado questionários específicos ou escalas para detecção de estresse nesses homens.

Talvez se tivéssemos aplicado escalas específicas para a detecção de ansiedade/ estresse e depressão na população, seria percebido um percentual maior de homens que se sentiam estressados com a nova configuração familiar (COHEN S, et al., 1983; COHEN S, 1986). Os estudos vêm mostrando que os parceiros e as gestantes consideram positiva a inclusão do homem no atendimento pré-natal.

Alves MI (2017) relatou em seu estudo que essa experiência foi positiva para $77,9 \%$ dos parceiros e para $78,8 \%$ das gestantes. Neste estudo, a participação foi espontânea, embora não tenha sido pesquisada diretamente a satisfação, considerou-se o resultado positivo, já que eles retornaram às consultas com os exames solicitados, receberam orientações e tratamento quando necessário. Outro dado relevante no estudo, foi a identificação de homens jovens (média 34 anos) já com sobrepeso, alteração de colesterol e triglicérides e patologias de base. Os valores de IMC (média 28,2) e de colesterol total (média 204) demonstram um aumento do risco de eventos cardiovasculares.

Devido à lipólise, muitos ácidos graxos livres (AGL) são liberados pelo tecido adiposo, associando-se à resistência insulínica. $O$ aumento dos triglicérides (TG) e AGL pode desencadear o depósito ectópico de TG no fígado e no miocárdio. Considerando a idade atual, se não houver mudanças nos hábitos (exercício físico, dieta equilibrada), fatalmente as complicações aparecerão, seja através de infarto ou AVC, frutos da síndrome metabólica iniciada precocemente (CALLEGARI SBM, et al., 2014). Portanto, a avaliação física e de exames complementares realizados identificaram alterações que devem ser abordadas de forma integrada pelo médico assistente, a fim de que complicações futuras sejam evitadas, pensando também no futuro dessa família que está sendo formada.

Os homens foram abordados na mesma consulta de sua esposa / companheira, sendo esclarecido do diagnóstico, dos cuidados necessários para controle de sua enfermidade e tratamento de forma imediata, sem uma série de marcações de consultas que muitas vezes afugentam o homem dos serviços de atenção básica (MINISTÉRIO DA SAÚDE, 2008).

A consulta de retorno foi agendada na clínica médica e nas especialidades apenas quando necessário. Essa abordagem eficaz otimiza o tempo e garante maior facilidade do atendimento ao homem, já que muitos ainda se consideram provedores e usam a carga de trabalho e horários das consultas como fatores incompatíveis para seu atendimento (MINISTÉRIO DA SAÚDE, 2008).

A realização de testes sorológicos para a detecção das infecções sexualmente transmissíveis de maior relevância (sífilis, HIV e hepatites) não apresentou resultados positivos em nenhum dos participantes. No Brasil a sífilis, apresentou alta de 1047\% entre 2005 e 2013 e os casos de notificação de sífilis congênita aumentaram em $135 \%$. A verdade é que não se entende porque ainda não se controlou o problema da sífilis congênita, já que o agente é conhecido com sensibilidade comprovada à penicilina, os exames de deteç̧ão estão disponíveis e a medicação tem baixo custo.

Segundo a FEBRASGO (2018), não se consegue compreender como alcançamos enorme sucesso no controle da infecção pelo HIV e apresentamos resultados tão desastrosos no controle da sífilis. Apesar desse aumento em nosso país, não se identificou nenhum caso de IST nas pacientes em regime de pré-natal e nem em seus parceiros sexuais, o que sugere que a população que frequenta nosso serviço está sendo bem orientada (MINISTÉRIO DA SAÚDE, 2019; BRASIL, 2019).

Um dado relevante é que temos o ambulatório (pré-concepcional) que assiste muitas pacientes antes da gestação e todas as medidas de controle de IST são também realizadas, com avaliação do colo uterino, tratamento do parceiro quando necessário e isso pode influenciar positivamente em nossos resultados. Após o tratamento de IST, os exames de controle são realizados na mulher e seu companheiro/marido, de forma a garantir que o tratamento foi eficaz e os homens são chamados para receberem orientações sobre a doença e formas de contágio. Sendo assim, quando identificadas, as patologias são tratadas previamente, de forma que a gravidez possa acontecer em um ambiente mais seguro para a gestante e bebê. 
Este estudo não mostrou benefício do pré-natal do parceiro para a saúde da gestante. Entretanto, os dados avaliados foram patologias orgânicas específicas como o descolamento prematuro de placenta, síndromes hipertensivas, diabetes gestacional, alterações de tireóide e outras. Talvez se a abordagem tivesse incluído dados emocionais (ansiedade, depressão) esses resultados pudessem ser diferentes, mas vale a pena destacar que mesmo em patologias com grande comprometimento emocional, como vômitos, não houve diferença entre os grupos.

Atualmente, existem várias escalas para identificar o estresse, bem como a depressão, mas, esses dados não foram avaliados, o que poderia ser considerado um fator limitante de nosso estudo, já que o suporte afetivo fornece conforto, segurança e confiança e na maioria das sociedades contemporâneas, a família tem este papel acolhedor e até de refúgio (WHOQOL, 1997; FLECK MP, et al., 2000; BAPTISTA MK, et al., 2010).

Importante ressaltar que mesmo que a participação dos parceiros sexuais no pré-natal não tenha alterado a ferquência de patologias maternas ou intercorrências clínicas que influenciariam no determinismo do parto, não se pode esquecer de seu papel emcional e subjetivo. Sua inclusão pode provocar reflexões que os ajudem os pais a se tornarem membros ativos no exercício da paternidade, desde a gestação. Essa visão mais atual, tira do homem o papel único de provedor material, o coloca como um membro ativo na formação emocional da família, participando de forma acolhedora no cuidado dos preparativos para a chegada do bebê, da sua companheira e, a seguir, nos cuidados do próprio bebê (CAVACANTI TRL e HOLANDA VR, 2019).

Esse estudo foi um piloto para que pudesse avaliar a forma de abordagem do homem durante o pré-natal por nossa equipe. Foi considerado um sucesso, já que os parceiros participaram desse momento, retornaram com exames e foram orientados sobre sua saúde e da sua companheira. Acredita-se que essa parceria (mulher/homem) seja capaz de reforçar os laços e compromissos, podendo impactar de forma positiva na saúde da grávida e na gestação refletindo durante o parto e puerpério, aumentando a segurança, autonomia e tranquilidade do casal.

\section{CONSIDERAÇÕES FINAIS}

Diante dos resultados obtidos nesse estudo, conclui-se que não há relação ou interferência do pré-natal do parceiro sobre a saúde da gestante e sobre o decorrer da gestação, considerando as doenças orgânicas pesquisadas. Entretanto, para a saúde do pai/parceiro, o estudo identificou sobrepeso e alterações metabólicas (colesterol / triglicérides) em alguns desses homens, permitindo a abordagem terapêutica e orientações para uma vida saudável.

\section{REFERÊNCIAS}

1. AGUIAR C, JENNINGS L. Impact of Male Partner Antenatal Accompaniment on Perinatal Health Outcomes in Developing Countries: A Systematic Literature Review. Matern Child Health J 2015; 19(9):2012-9.

2. ALVES MI. A inclusão do parceiro na assistência pré-natal. Dissertação. (Mestrado em Ginecologia e Obstetrícia). Universidade de São Paulo. Ribeirão Preto. 2017; 138p.

3. BAPTISTA MK, et al. Estudo Psicométrico de Escalas de Depressão (EDEP e BDI) e o Inventário de Percepção de Suporte Familiar (IPSF). Psicol. pesq. 2010; 4(1): 65-73.

4. BENAZZI AST et al. Pré-natal masculino: um novo olhar sobre a presença do homem. Rev Pol Publ. 2011; 15(2):327333.

5. BRASIL. Boletim Epidemiológico HIV / Aids. Ed. do Ministério da Saúde, Brasília.

6. BRASIL. MINISTÉRIO DA SAÚDE. Guia do pré-natal do parceiro para profissionais de saúde. Ed. do Ministério da Saúde, Brasilia; 2016. 56 p.

7. CALLEGARI SBM, et al. Obesidade e fatores de risco cardiometabólicos durante a gravidez. Rev Bras Ginecol e Obstet. 2014;36(10):449-55.

8. CARDOSO VEPS, et al. A Participação do Parceiro na Rotina Pré-Natal Sob a Perspectiva da Mulher Gestante. Res Fundam Care. 2018;10(3):856-62.

9. CAVALCANTI TRL, HOLANDA VR. Participação paterna no ciclo gravídico-puerperal e seus efeitos sobre a saúde da mulher. Enferm. Foco. 2019; 10 (1): 93-98

10. COFEN. CONSELHO FEDERAL DE ENFERMAGEM. Consulta pré-natal do parceiro é incluída nos procedimentos do SUS. 2017.

11. COHEN S, et al. A global measure of perceived stress. J Health Soc Behav. 1983; 24:385-96. 
12. COHEN S. Contrasting the Hassles Scale and the Perceived Stress Scale: Who's Really Measuring Appraised Stress? Am Psychol. 1986; 41:716-18.

13. CORTEZ MB, et al. Profissionais de saúde e o (não) atendimento ao homem-pai: análise em representações sociais. Psicol em Estud. 2016;21(1):53-63.

14. DUARTE G. Extensão da assistência pré-natal ao parceiro como estratégia de aumento da adesão ao pré-natal e redução da transmissão vertical de infecções. Rev Bras Ginecol Obs. 2007;29(4):171-4.

15. FÁBIO SV. Pré-natal do parceiro como estratégia para redução da transmissão vertical das doenças sexualmente transmissíveis e melhora dos indicadores de saúde perinatal. Dissertação (Mestrado em Ginecologia e Obstetrícia). Universidade de São Paulo. Ribeirão Preto. 2016; 94p.

16. FARIAS SMC et al. Caracterização dos sintomas físicos de estresse na equipe de pronto atendimento. Rev Esc Enferm USP 2011; 45(3):722-9

17. FEBRASGO. Sífilis na gravidez. 2018. Disponível em https://www.febrasgo.org.br/pt/noticias/item/700-sifilis-nagravidez. Acesso em 30/09/2020.

18. FLECK MP. O instrumento de avaliação de qualidade de vida da Organização Mundial da Saúde (WHOQOL-100): características e perspectivas. Cienc Saude Coletiva. 2000;5(1):33-8.

19. FREITAS WMF, et al. Paternidade: responsabilidade social do homem no papel de provedor. Rev Saude Publica. 2009;43(1):85-90.

20. HENZ GS, et al. A inclusão paterna durante o pré-natal. Rev enferm atenção saúde [Internet]. $2017 ; 8$ : 52-66.

21. HOLANDA SM et al. Influência da participação do companheiro no pré-natal: satisfação de primíparas quanto ao apoio no parto. Texto Contexto Enferm, 2018; 27(2):1-10.

22. HORTA HHL et al. Pré-natal do parceiro na prevenção da sífilis congênita. 2017; 20(4): 623-627.

23. MARGIS R. Relação entre estressores, estresse e ansiedade. Rev. psiquiatr. Rio Gd. Sul. $2003 ; 25$ (1):65-74.

24. MINISTÉRIO DA SAÚDE. BRASIL. Boletim Epidemiológico - Sífilis. 2019. 43p.

25. MINISTÉRIO DA SAÚDE. BRASIL. Política Nacional de Atenção à Saúde do Homem. Princípios de Diretrizes. Disponível em http://bvsms.saude.gov.br/bvs/publicacoes/politica_nacional_atencao_saude_homem.pdf. Acesso em 19/11/2020.

26. NOGUEIRA JFDF, FERREIRA M. O envolvimento do pai na gravidez/parto e a ligação emocional com o bebê. Rev Enf Ref 2012;3(8):57-66.

27. PORTO CC; PORTO AL. Semiologia Médica. 2019. 1360p.

28. WHOQOL Group. World Health Organization. WHOQOL: measuring quality of life. Geneva: WHO; 1997 (MAS/MNH/PSF/97.4). 